\title{
Intraoperative Localisation of Ectopic Parathyroid Adenoma with a Magnetic Marker
}

\author{
C Clement $^{1,2^{*}}$, DM den Hoed ${ }^{1}$ and P Jansen ${ }^{1}$ \\ ${ }^{1}$ Department of General Surgery, ETZ, The Netherlands \\ ${ }^{2}$ Department of General Surgery, KU Leuven, Belgium
}

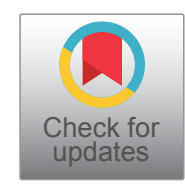

\begin{abstract}
Background: Primary hyperparathyroidism is a common clinical condition but identifying hyperfunctioning parathyroid glands can prove a diagnostic challenge preoperatively. Intraoperatively a systemic exploration might be warranted to localise the affected tissue, especially when the glands are in an ectopic position. Minimally invasive parathyroidectomy is an effective and safe surgical technique. To facilitate a focused parathyroidectomy for an ectopic parathyroid gland effective use of pre- or intraoperative ultrasound has been described but has several limitations.

Case report: We present a patient with primary hyperparathyroidism due to an ectopic parathyroid adenoma located between the left carotid artery and the hyoid bone. A magnetic marker was used to localise this ectopic gland intraoperatively and a parathyroidectomy was performed. Histology confirmed a parathyroid adenoma. The patient could be discharged without postoperative complications and normalisation of parathyroid hormone levels.
\end{abstract}

Conclusion: A magnetic marker can be a safe and effective method to localise ectopic parathyroid glands during focused parathyroidectomy. Further proof of concept studies seems to be warranted.

\section{Keywords}

Magnetic marker, Parathyroid adenoma, Parathyroid gland

\section{Introduction}

Up to $90 \%$ of primary hyperparathyroidism cases are attributable to a single parathyroid adenoma, making parathyroidectomy the golden standard in curative treatment [1]. Ectopic parathyroid glands are found in $6-16 \%$ of patients $[2,3]$. Until recently, Tecnetium-99-sestamibi scintigraphy (MIBI-scan) was considered standard, but several studies have shown high-resolution cervical ultrasound is accurate as the only diagnostic modality for identifying abnormal glands. (9398\%) [4-6]. However, ectopic glands behind bony structures can be missed and other diagnostic modalities may prove useful. Identifying these glands preoperatively is necessary for focused parathyroidectomy, preferred to bilateral neck exploration due to less morbidity [1,5].

Magnetic seeds, such as Magseed ${ }^{\circledR}$ (Endomagnetics Ltd, CE-registered and FDA-cleared) or Pintuition ${ }^{\circledR}$ (Sirius Medical, CE-registered and FDA-cleared), have been shown to be safe in other applications, such as localising non-palpable breast lesions as well as lymph nodes $[7,8]$. To our knowledge this is the first report of intraoperative localisation of ectopic parathyroid adenoma using a magnetic marker.

\section{Case Presentation}

A 64-year-old man was referred to the Internal Medi- cine outpatient clinic with hypercalcaemia. Previous medical history included 40 pack years. In a work-up for suspected sarcoidosis hypercalcaemia was detected of $2.93 \mathrm{mmol} / \mathrm{L}$ (normal range: $2.20-2.65 \mathrm{mmol} / \mathrm{L}$ ). After treatment with aminopropylidine diphosphonate (APD) infusion and start of thiazide diuretic calcium diminished to $2.83 \mathrm{mmol} / \mathrm{L}$. Desquamative interstitial pneumonia was diagnosed after ruling out sarcoidosis on pathologic examination of lung tissue.

Subsequently, primary hyperparathyroidism was diagnosed with an increased parathyroid hormone of $12.4 \mathrm{pmol} / \mathrm{L}$ (normal range: $1.9-8.4 \mathrm{pmol} / \mathrm{L}$ ), in absence of $25-\mathrm{OH}$ Vitamin $D$ deficiency $(53 \mathrm{nmol} / \mathrm{L}$, normal range $>50 \mathrm{nmol} / \mathrm{L}$ ). Presenting symptoms included abdominal pain and constipation.

*Corresponding author: C Clement, Department of General Surgery, ETZ, Hilvarenbeekseweg 60, 5022 GC Tilburg, The Netherlands; Department of General Surgery, KU Leuven, Belgium

Accepted: May 21, 2021

Published online: May 23, 2021

Citation: Clement C, Hoed DM, Jansen P (2021) Intraoperative Localisation of Ectopic Parathyroid Adenoma with a Magnetic Marker. J Surgical Endocrinol 3(1):77-80 
Citation: Clement C, Hoed DM, Jansen P (2021) Intraoperative Localisation of Ectopic Parathyroid Adenoma with a Magnetic Marker. J Surgical Endocrinol 3(1):77-80

Osteoporosis was detected on dual-energy X-ray absorptiometry with a T-score of -3.2 of bone mineral density in the

lumbar spine.

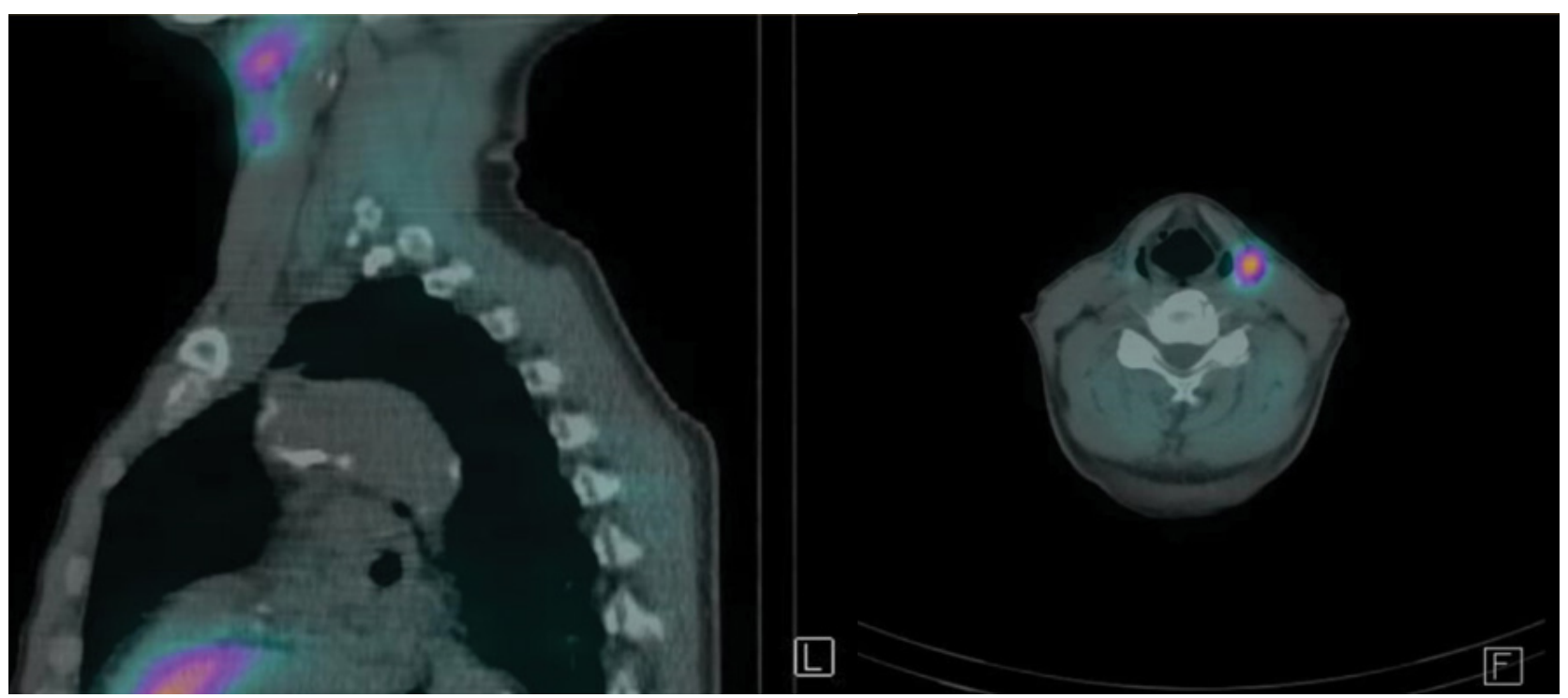

Figure 1: MIBI scan: Sagittal and coronal view.
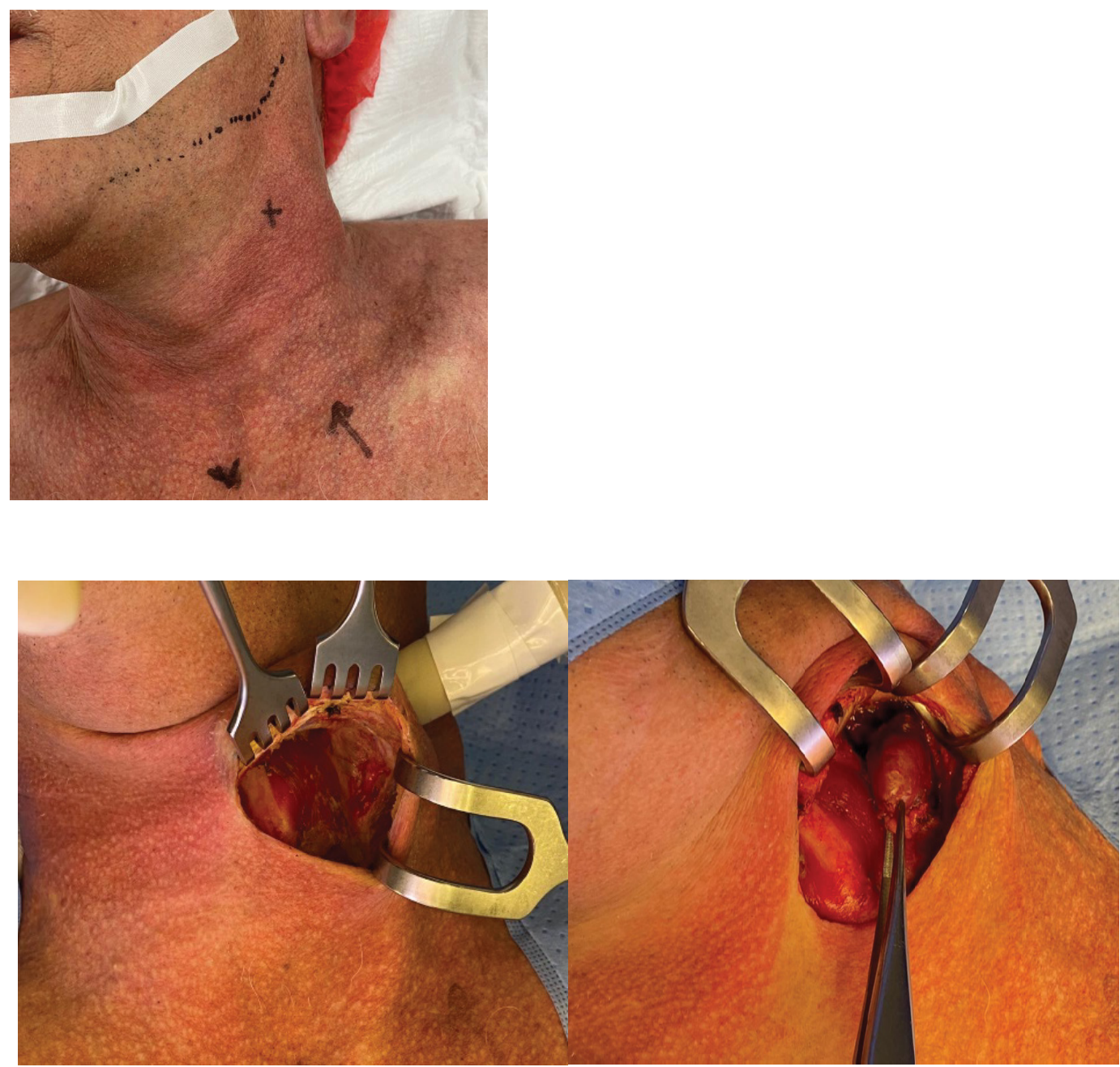

Figure 2: (A) Preoperative marking of the sternoclavicular notch, inferior border of the mandible and a marked " $x$ " where signal of Pintuition was detected; (B) Intraoperative view: Probe detecting magnetic implant lateral of strap muscles; (C) Adenoma. 
Clinical examination of the thyroid and cervical lymph nodes was unremarkable. During diagnostic work-up retention of technetium was observed proximally of the left thyroid lobe on MIBI-scan, matching an enlarged tissue abnormality of $27 \mathrm{~mm}$ by $12 \mathrm{~mm}$ at level 2 (left), between the carotid artery and the hyoid bone, on ultrasound. (Figure 1) Nine days preoperatively a $3 \mathrm{~mm}$ magnetic marker, Pintuition ${ }^{\circledR}$ Seed, was placed in the ectopic parathyroid gland with ultrasound guidance using a $14 \mathrm{G}$ needle.

A focused parathyroidectomy was performed. After intubation the patient was placed in supine position with the head in hyperextension. With the probe detecting the magnetism of the marker, the gland was localised, distance to the probe measured and marked on the skin (Figure 2). An incision was made at the cervical midline at the level of the thyroid notch and the platysma was opened. Using the probe, the suspected adenoma was identified laterally of the omohyoid muscle and medially of the external carotid artery (Figure 3). The location of the marker within the resection specimen was confirmed by the probe. Operation time was 36 minutes. Frozen section of the tissue confirmed the tissue to be parathyroid tissue. Definitive histopathologic examination confirmed a parathyroid adenoma.

One day postoperatively the patient was discharged without any complications. Blood results showed values within the normal range for albumin $(38 \mathrm{~g} / \mathrm{L})$, calcium $(2.37 \mathrm{mmol} / \mathrm{L})$ and a $50 \%$ reduction of PTH compared to preoperatively (preoperatively $6.5 \mathrm{pmol} / \mathrm{L}$ and postoperatively $3.5 \mathrm{pmol} / \mathrm{L}$ ). Further follow-up was performed through the Endocrinology outpatient clinic.

Written consent from the patient for this article was obtained.

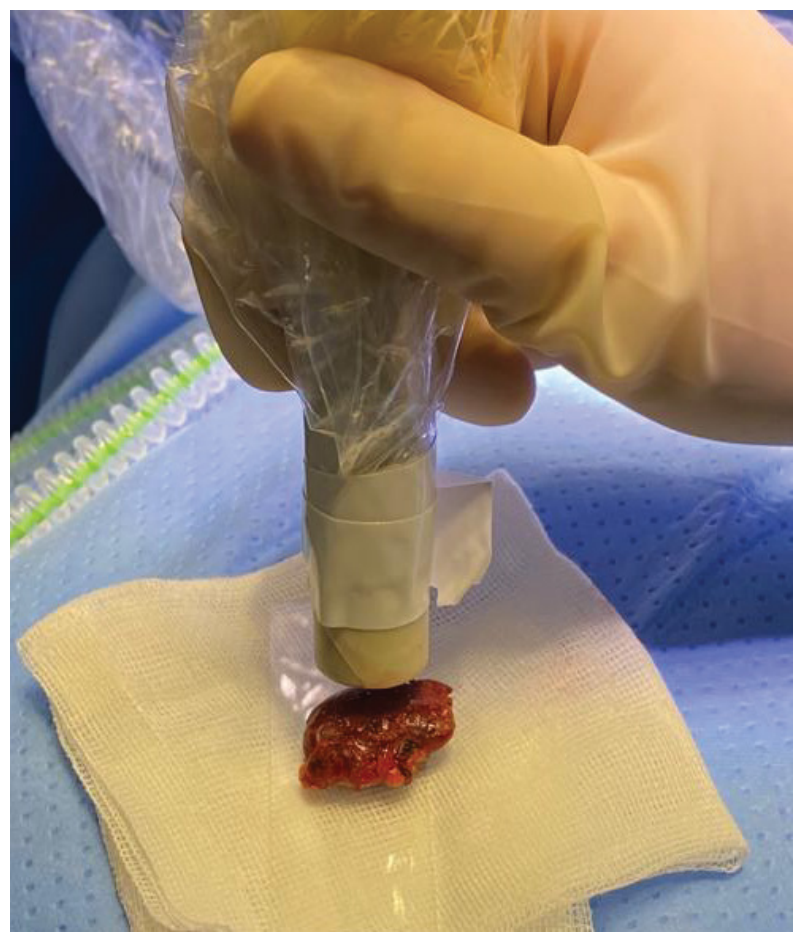

Figure 3: Verifying retrieval of Pintuition in adenoma.

\section{Discussion}

Parathyroidectomy is more cost-effective than best medical treatment for primary hyperparathyroidism, which is undertreated in the majority of patients $[9,10]$. In this symptomatic patient the indication for parathyroidectomy is apparent according to the guidelines of the Endocrine Society [11]. An ectopic localisation of an adenoma is common due to the embryological origin of the parathyroid gland $[3,12,13]$. Imaging is not required for diagnosis of primary hyperparathyroidism but is a necessary aide for surgery. Focused parathyroidectomy has been proven to be associated with shorter procedure times, lower rate of hypocalcaemia and recurrent laryngeal nerve injury $[1,5]$. Optimal localisation of the gland is therefore essential but can be challenging pre- and intraoperatively. Several techniques have been introduced as a road map for surgery, but with differences in sensitivity and costs [14]. Although a MIBI-scan was performed, in this case its use and cost-effectiveness are debatable. A high-resolution cervical ultrasound is a cost-effective and sensitive technique to localise an ectopic parathyroid adenoma, however intraoperatively not useful to identify a parathyroid adenoma after incision. Minimally invasive radioguided parathyroid surgery is a feasible technique for ectopic glands, however it is controversial and has logistical limitations related to the use of radioactive products [15-17]. Magnetic markers can be placed using ultrasonography. Distance-based measurements can accurately localise the parathyroid gland during surgery. With limited contraindications for its usage, allowing decoupling of radiology and surgical schedules and no need for radioactivity, magnetic markers are a clinician and patient-friendly option [8]. Absolute contra-indication for its use is a pacemaker in proximity of the marker. Relative contra-indications consist of metallic interference of nearby surgical instruments or the need for Magnetic Resonance Imaging (MRI) due to scattering on imaging [7]. For other applications magnetic markers seem to be cost-effective and few adverse events have been reported [18]. No adverse events concerning the magnetic marker, nor postoperative complications were reported in this patient.

While surgical experience and anatomical knowledge are key to an effective focused parathyroidectomy, a magnetic marker can be a useful adjunct intraoperatively to confirm the identification of the gland. This may reduce the need for intraoperative PTH monitoring and frozen section of the specimen in the future [6]. Thus, this application can reduce operating time and costs, while improving patient outcome.

\section{Conclusion}

Ectopic parathyroid glands can prove a diagnostic challenge and may warrant a systemic exploration. A magnetic marker can be a safe and effective method to localise ectopic parathyroid glands during focused parathyroidectomy reducing operating time and risk of complications. This is the first case report to our knowledge describing successful application of a magnetic marker.

\section{References}

1. Singh Ospina NM., Rodriguez-Gutierrez R, Maraka S, et al. (2016) 
Outcomes of parathyroidectomy in patients with primary hyperparathyroidism: A systematic review and meta-analysis. World Journal of Surgery 40: 2359-2377.

2. Roy M, Mazeh H, Chen H, et al. (2013) Incidence and localization of ectopic parathyroid adenomas in previously unexplored patients. World Journal of Surgery 37: 102-106.

3. Taterra D, Wong LM, Vikse J, et al. (2019) The prevalence and anatomy of parathyroid glands: A meta-analysis with implications for parathyroid surgery. Langenbeck's Archives of Surgery 404: 63-70.

4. Butt HZ, Husainy MA, Bolia A, et al. (2015) Ultrasonography alone can reliably locate parathyroid tumours and facilitates minimally invasive parathyroidectomy. Annals of the Royal College of Surgeons of England 97: 420-424.

5. Schneider R, Hinrichs J, Meier B, et al. (2019) Minimally invasive parathyroidectomy without intraoperative PTH performed after positive ultrasonography as the only diagnostic method in patients with primary hyperparathyroidism. World Journal of Surgery 43: 1525-1531.

6. Yeung M (2020) Parathyroidectomy without the utilisation of iPTH: The gold standard is still a good operation-how understanding the anatomy and a simple US can help. World Journal of Surgery 44: 622-624.

7. Mayo RC, Kalambo MJ, Parikh JR (2019) Preoperative localization of breast lesions: Current techniques. Clinical Imaging 56: 1-8.

8. Bessems M, van Breest Smallenburg V, van Bebber I, et al. (2021) Safety and performance of sirius pintuition; a novel wire-free and non-radioactive localization system for breast cancer surgery. European Journal of Surgical Oncology 47: e1.

9. Wilhelm SM, Wang TS, Ruan DT, et al. (2016) The American association of endocrine surgeons guidelines for definitive man- agement of primary hyperparathyroidism. JAMA Surgery 151: 959-968.

10. Seib CD, Suh I, Meng T, et al. (2021) Patient factors associated with parathyroidectomy in older adults with primary hyperparathyroidism. JAMA Surg 156: 334-342.

11. Bilezikian JP, Brandi ML, Eastell R, et al. (2014) Guidelines for the management of asymptomatic primary hyperparathyroidism: Summary Statement from the Fourth International Workshop. J Clin Endocrinol Metab 99: 3561-3569.

12. Akerström G, Malmaeus J, Bergström R (1984) Surgical anatomy of human parathyroid glands. Surgery 95: 14-21.

13. Musters L, van Ginhoven TM, Smit C (2014) Exceptional localisation of an ectopic parathyroid adenoma at the level of the hyoid bone. BMJ Case Reports 2014: bcr2013200557.

14. Lubitz CC, Stephen AE, Hodin RA, et al. (2012) Preoperative localization strategies for primary hyperparathyroidism: An economic analysis. Ann Surg Oncol 19: 4202-4209.

15. Desiato V, Melis M, Amato B, et al. (2016) Minimally invasive radioguided parathyroid surgery: A literature review. International Journal of Surgery 28: S84-S93.

16. Chen H, Mack E, Starling JR, et al. (2005) A comprehensive evaluation of perioperative adjuncts during minimally invasive parathyroidectomy: Which is most reliable? Ann Surg 242: 375-383.

17. Inabnet III WB, Dakin GF, Haber RS, et al. (2002) Targeted parathyroidectomy in the era of intraoperative parathormone monitoring. World Journal of Surgery 26: 921-925.

18. Lindenberg M, van Beek A, Retèl V, et al. (2020) Early budget impact analysis on magnetic seed localization for non-palpable breast cancer surgery. PLoS One: 15. 